\title{
Multiple Advisors with Reputation*
}

\author{
Junghun Cho \\ Texas A\&M University
}

October 24, 2005

\begin{abstract}
This paper examines the existence of good and bad reputation effects in a two period cheap talk model where the decision maker receives messages from two advisors. I assume that each advisor perfectly knows the type of the other advisor, but his signal about the state of the world is imperfect. Strong reputational concern makes the good advisor sometimes tell a lie in the first period regardless of the type of the other advisor. It is shown that the presence of the other advisor does affect the message sent by an advisor. The good advisor has a greater incentive to tell a lie when he knows that the other advisor is bad rather than good. If each type of advisor considers his second period as sufficiently more important, it is better for the decision maker to have a single advisor.
\end{abstract}

\section{Introduction}

Many decisions are made after obtaining advises from others. Before going to the movie or buying new computer, we usually seek advises from our friends each of whom has more private information than us. In many situations, there are some advisors who are biased towards suggesting a particular strategy when the information is conveyed by cheap talk. Before going to the college, we ask advises from many people in order to choose the proper field or the proper college for us. Some advisors are biased towards suggesting a particular field or college because they believe that their suggested field or college can increase the chances of obtaining a job in the future regardless of either our interests or market conditions. As a more specific example, consider a market expert who is not biased towards suggesting any

\footnotetext{
*I am grateful to Rajiv Sarin for many valuable comments and helpful discussions.
} 
particular pricing policy and the manager of a firm who is also not biased towards using a particular policy. If the manager of the firm has to choose an action under uncertainty, he seeks the advice from an expert. Because of imperfect information about the type of the expert, the manager of the firm may believe that the expert can be aggressive market expert who is biased towards suggesting the aggressive pricing policy. Consider the case where the expert concludes that an aggressive pricing policy, a price cut, is needed. If the strong reputational concern of the market expert (who has the same preferences as the manager of the firm) makes him suggest defensive strategy because he does not want to be perceived as an aggressive expert, the manager of the firm loses information. The incentive to tell a lie exists because the expert wants the manager to believe what he will suggest next time. In contrast to the result of informative cheap talk, even if the market expert has the same preferences as the manager of the firm, the strong reputational concern of the expert leads to the loss of information. If the manager of the firm believes that he may not obtain the correct information from a single market expert, he may try to seek advice from an additional expert under the belief that the presence of the other expert may change the message of the first expert, and vice-versa.

The first question is whether the existence of the other expert changes the message of one expert when the types of experts are mutually known. If so, I can examine whether the existence of the other expert affects the possibility that the first expert will tell a lie or the truth. Although the manager of the firm obtains additional information when he has two experts compared to the case where he has only one expert, the likelihood of telling a lie may increase because of the existence of the other expert. The next question is whether the manager of the firm can benefit if he takes advice from an additional expert when each expert knows the type of the other expert and both experts send their message simultaneously.

I consider a two period cheap talk model with two advisors to the decision maker. The advisors can be of tow types - good or bad. Good advisor is assumed to have exactly the same preferences as the decision maker, while the bad advisor has a payoff bias towards one of the two actions available to the decision maker. Each advisor observes a private signal regarding the state of the world, 0 or 1 , and then sends a message ( 0 or 1$)$ to the decision maker who does not have any prior information regarding the state of the world. The decision maker then chooses an action which can affect all players' payoffs. The real state of the world is revealed after the decision 
maker takes an action. The decision maker then updates his belief about the type of each advisor taking into account the message sent by each advisor and the real state of the world. The same stage game is repeated in the next period with the decision maker again consulting the same advisors. In the example, the expert who has the same preferences as the manager of the firm is referred to the good expert while the expert who is biased towards suggesting the aggressive pricing policy is termed as the bad expert. After obtaining the messages from both experts, the manager of the firm determines the price as an action.

The term "good reputation effect" refers to the bad advisor sending a truthful message after receiving a signal that the state of the world is the one he is not biased towards. The term "bad reputation effect" involves the good advisor sending an untrue message after receiving a signal that the state of the world is the one the bad advisor is biased towards. It is important to note that the both of these effects arise from the reputational concern of the advisor to be perceived as a good advisor by the decision maker.

If each advisor has perfect information about the state of the world, I cannot determine the existence of a bad reputation effect. This paper shows that both advisors have reputational incentive to send the message the bad advisor is not biased towards if each advisor has imperfect private information. In the above example, suggesting a defensive pricing policy can increase the reputation of each type of expert even if the real state of the world demands an aggressive pricing policy. This is because the manager of the firm knows the experts have imperfect information regarding the state of the world, and suggesting a defensive pricing policy is the way for good expert to distinguish himself from bad expert.

If the bad advisor considers his second period as sufficiently more important, he tells the truth even if he has loss in current payoff when the signal is the one he is not biased towards. When the signal is the one the bad advisor is biased towards, the good advisor tells a lie if he considers his second period as sufficiently more important. There is a greater (lesser) incentive for the good advisor to tell a lie when the other advisor is bad (good). By using numerical example, it is shown that the existence of the other advisor reinforces the bad reputation effect. By comparing the expected payoff of the decision maker when he has two advisors with that of the decision maker when he has a single advisor, I find that it is better for the decision maker 
only to have a single advisor if each type of advisor considers his second period as sufficiently more important. If the decision maker is so skeptical and so believes that each type of advisor considers the second period as sufficiently more important, i.e. if he believes each type of advisor sends the message the bad advisor is not biased towards in the first period, he is better off of asking an advice from a single advisor.

In cheap talk models, the question is when there exist equilibria where the cheap talk from the sender (the advisor) to the decision maker is informative. ${ }^{1}$ To make cheap talk informative, three necessary conditions are needed. The different sender types have different preferences over the actions of the decision maker, the decision maker prefers the different actions depending on the sender's type, and the decision maker's preferences over actions cannot be completely opposed to the sender's (Gibbons, 1992). Crawford and Sobel (1982) examine the strategic information transmission from one advisor to the decision maker that satisfies these three conditions. By characterizing partially pooling equilibria, they find that more communication is possible when the preferences of the two players are more closely aligned, and the perfect information is conveyed when two players have the same preferences. Compared to the single advisor's cheap talk model, it is also possible to consider the welfare effect of the decision maker in multiple advisors' cheap talk model. Krishna and Morgan (2001) examine a one period cheap talk model with two advisors who send messages sequentially. By comparing the payoff of the decision maker when he has a single advisor with that of the decision maker when he has two advisors, they show that it is never beneficial to consult both advisors if both advisors are biased in the same direction. However, if the two advisors are biased in opposite directions, it is always beneficial to consult both advisors. Since they examine one period model, each advisor has no reputational concern when he sends the message.

Consultants will most likely be concerned about their reputation if they are engaged in a repeated interaction with the decision maker. ${ }^{2}$ Sobel (1985) considers a finite cheap talk game where there is a single advisor who can be

\footnotetext{
${ }^{1}$ Battaglini (2002, 2004) and and Levy and Razin (2004) consider multidimensional cheap talk models. In Olszewski (2003), the decison maker also has private information regarding the state of the world. Park (2004) finds the conditions that make cheap talk informative in infinitely repeated cheap talk game.

${ }^{2}$ Kreps and Wilson (1982) and Milgrom and Roberts (1982) developed the theoretical model of the reputation where long run player meets a sequence of the short run players.
} 
one of the two types - enemy (an informed advisor with completely opposing interests to the decision maker) or friend (with identical interests to the decision maker), and finds that there is an incentive for an enemy to behave like a friend in order to increase his reputation. ${ }^{3}$ Even if he considers the good reputation effect (as a term of my paper), the enemy is different as the bad advisor because the bad advisor is the person who is biased towards suggesting any particular strategy. Such as the bad advisor sometimes tells the truth to increase his reputation, the good advisor who has the same preferences as the decision maker sometimes tells a lie to increase his reputation in the case where there is one advisor to the decision maker. Morris (2001) considers a two period cheap talk model with one advisor having imperfect information regarding the state of the world. In equilibrium condition, an advisor has reputational incentive to send the particular message the bad advisor is not biased towards in order to separate his type from the bad type regardless of the signal. Especially if an advisor considers the second period as sufficiently more important, no information is conveyed in the first period. ${ }^{4}$ This paper extends Morris' model by considering multiple advisors each of whom knows the type of the other advisor. Comparing the results, I can examine the effect of the presence of the other advisor and the welfare of the decision maker.

In section 2, using a two period cheap talk model, I will examine the conditions required for the existence of both good and bad reputation effects when each advisor has imperfect information regarding the state of the world and has perfect information regarding the type of the other advisor. I will examine the welfare effect of the decision maker in section 3 .

\section{Model}

I consider a two period cheap talk model where the decision maker has two advisors, each of whom knows the type of the other advisor. It is assumed that the decision maker does not know the state of the world and seeks the advice of both advisors. After receiving private signal regarding the state

\footnotetext{
${ }^{3}$ In Ottaviani and Sorensen (2004), the real state of the world is revealed to the decision maker before the decision maker chooses his action. The action of the decision maker is evaluation of the advisor by comparing the message to the real stae of the world.

${ }^{4}$ Ely and Valimaki (2003) consider a model where long lived mechanic interacts with a sequence of short run motorists. Bad reputation effect can emerge if there is imperfect information to the motorists regarding the type of the mechanic.
} 
of the world, both advisors simultaneously send a costless message to the decision maker.

The state of the world in period $i$ is $\omega_{i} \in W=\{0,1\}$ for $i=1,2$. Each state is equally likely, i.e. $P\left(\omega_{i}=0\right)=\frac{1}{2}=P\left(\omega_{i}=1\right.$ ). The advisor $j$ (for $j=1,2)$ observes a signal $S_{i}^{j}$ regarding the state of the world in period $i$. The decision maker does not know the type of advisors. The decision maker believes the advisor $j$ is good with probability $\lambda_{i}^{j}$ in each period $i$. With probability $1-\lambda_{i}^{j}$, the decision maker believes the advisor $j$ is bad. After observing the signal, advisor $j$ sends the message $m_{i}^{j}$ to the decision maker. After obtaining messages from both advisors, the decision maker chooses his action $a_{i} \in R$ which affects all players' payoffs. After the decision maker chooses his action, the state of the world in period $i$ is revealed publicly. The message in the first period plays the additional role of changing the belief of the decision maker about the type of the advisor. After observing the state of the world in the first period, the decision maker updates the belief that the advisor $j$ is good, $\lambda_{2}^{j}=\lambda_{2}^{j}\left(\lambda_{1}^{j}, m_{1}^{j}, \omega_{1}\right)$, where $\lambda_{1}^{j}$ is prior belief of the decision maker that advisor $j$ is good.

The utility function of the decision maker and the good advisor is assumed to be $-\left(a_{i}-\omega_{i}\right)^{2}$ in each period $i$. Since it is assumed that the decision maker does not know the state of the world, the decision maker chooses the action $a_{i}$ which is the probability that the state of the world is 1 given messages from both advisors. The utility function of the bad advisor is assumed to be $a_{i}$ in each period $i$. The payoff obtained by the bad advisor in period $i$ is the greatest if the decision maker chooses action 1. Each type of advisor may put different weights on each period. The total utility of advisor $j$, if he is of the good type, is

$$
-x_{1}^{j}\left(a_{1}-\omega_{1}\right)^{2}-x_{2}^{j}\left(a_{2}-\omega_{2}\right)^{2},
$$

where $x_{1}^{j}$ and $x_{2}^{j}$ denote the weights on the payoffs in the first period and in the second period respectively. The total utility of advisor $j$, if he is of the bad type, is

$$
y_{1}^{j} a_{1}+y_{2}^{j} a_{2},
$$

where $y_{1}^{j}$ and $y_{2}^{j}$ denote the weights on the payoffs in the first period and in the second period respectively. Since both good and bad reputation effects are determined by the weight on the payoff in the first period, it is assumed that the sum of the weight in each period is 1 . 
I will use backward induction to solve the model. I first solve for the action taken by the decision maker after receiving messages from both advisors during the second period. Knowing the decision maker's action for each message in the second period, I am able to determine the value function for each type of the advisor. While sending the message in the first period, the advisors consider not only their payoffs in the first period, but also the expected payoffs in the second period which are determined by the value function.

If each advisor observes a perfect signal regarding the state of the world, each advisor has no reputational incentive to tell a lie in the first period. When the state of the world in the first period is revealed as 1 , I cannot guarantee the updated belief of the decision maker that an advisor is good increases if advisor sends the message 0 . It is because the decision maker will know that an advisor is a liar and will not believe what he will say in the next period. Each advisor has an incentive to send the message truthfully in the first period if he wants the decision maker to believe what he will say in the next period. The bad reputation effect cannot be observed if each advisor has perfect information regarding the state of the world.

Consider the case where advisor $j$ observes an imperfect signal $S_{i}^{j}$ in period $i$ regarding the state of the world $\omega_{i}$. Let $\gamma$ denote the probability that the state of the world is the same as the signal received by each advisor, i.e. $\gamma=P\left(S_{i}=\omega_{i}\right)$. The signal is imperfect but informative, such that $\frac{1}{2}<\gamma<1$. Since it is assumed that each advisor has the same ability to obtain the signal regarding the state of the world, the same probability that the state of the world is the same as the signal received by an advisor is applied to each advisor.

\subsection{Second Period}

There is always babbling equilibrium in cheap talk model. Except babbling equilibrium where the decision maker learns nothing about the type of the advisor and the state of the world, this model examines the informative equilibrium of the cheap talk model. In any informative equilibrium in the second period, since this is the last period each advisor does not consider his reputation. The advisor $j$, if he is of the good type, sends the message $m_{2}^{j}=k$ when his signal in the second period is $k$ for $k=0$ or 1 . If the advisor $j$ is of the bad type, regardless of the signal he sends the message $m_{2}^{j}=1$. 
In order to determine the value function in any informative equilibrium, the probability that the state of the world in the second period is 1 given messages from the advisors must be calculated. As discussed before, the decision maker knows that the bad advisor will never send the message 0 in the second period. The probability that the state of the world is 1 given the message 0 from both advisors in the second period is given by

$$
P_{0,0}^{2,1}=\frac{(1-\gamma)^{2}}{\gamma^{2}+(1-\gamma)^{2}}
$$

where $P_{m_{i}^{1}, m_{i}^{2}}^{i, 1}$ represents the probability that the state of the world is 1 in period $i$ given the message of the first advisor $m_{i}^{1}$ and the message of the second advisor $m_{i}^{2}$. Since the action of the decision maker is the probability that the state of the world is 1 given messages from both advisors, the decision maker chooses action $P_{0,0}^{2,1}$ when he receives the message 0 from both advisors.

If the decision maker receives $m_{2}^{1}=0$ and $m_{2}^{2}=1$ in second period, he will be able to infer that the first advisor is good but the second advisor may be of either good or bad type. The belief of the decision maker that the state of the world is 1 becomes

$$
P_{0,1}^{2,1}=\frac{(1-\gamma)\left(1-\lambda_{2}^{2}+\gamma \lambda_{2}^{2}\right)}{1-\left(1-2 \gamma+2 \gamma^{2}\right) \lambda_{2}^{2}}
$$

and the decision maker chooses action $P_{0,1}^{2,1}$ when he receives the message 0 from the first advisor and 1 from the second advisor.

If the decision maker receives the message 1 from the first advisor and the message 0 from the second advisor, the probability that the state of the world in the second period is 1 becomes

$$
P_{1,0}^{2,1}=\frac{(1-\gamma)\left(1-\lambda_{2}^{1}+\gamma \lambda_{2}^{1}\right)}{1-\left(1-2 \gamma+2 \gamma^{2}\right) \lambda_{2}^{1}}
$$

and the decision maker chooses action $P_{1,0}^{2,1}$.

When the decision maker receives the message 1 from both advisors, then he believes both advisors may be good, both may be bad, or only one advisor may be good. The probability that the state of the world is 1 will be

$$
P_{1,1}^{2,1}=\frac{1-(1-\gamma)\left(\lambda_{2}^{1}+\lambda_{2}^{2}\right)+(1-\gamma)^{2} \lambda_{2}^{1} \lambda_{2}^{2}}{2-\lambda_{2}^{1}-\lambda_{2}^{2}+\left(1-2 \gamma+2 \gamma^{2}\right) \lambda_{2}^{1} \lambda_{2}^{2}}
$$


and the decision maker chooses action $P_{1,1}^{2,1}$ when he receives the message 1 from both advisors.

The value function of the bad advisor is determined using the action of the decision maker given that the message of bad advisor is 1 . Since each advisor knows the type of the other advisor, let's start to calculate the value function of the bad advisor when the bad advisor knows the other advisor is of the good type. Suppose that the first advisor is of the bad type. When the bad advisor knows that the other advisor is good, the value function for the bad advisor is

$$
v_{B G}^{1}\left[\lambda_{2}^{1}, \lambda_{2}^{2}\right]=y_{2}^{1} a_{2}=\frac{1}{2} y_{2}^{1}\left(P_{1,0}^{2,1}+P_{1,1}^{2,1}\right) .
$$

It is because the bad advisor sends the message 1 regardless of the signal and the good advisor sends the message which is the signal he observes in each state of the world. In each state of the world, each advisor obtains the correct signal with probability $\gamma$. When the bad advisor knows that the other advisor is also of the bad type, the value function of the bad advisor is

$$
v_{B B}^{1}\left[\lambda_{2}^{1}, \lambda_{2}^{2}\right]=y_{2}^{1} a_{2}=y_{2}^{1} P_{1,1}^{2,1} .
$$

It is because both bad advisors send the message 1 regardless of signal.

The value function of the good advisor is determined using the action of the decision maker in each state of the world. Suppose that the first advisor is of the good type. The value function for the good advisor is

$$
\begin{aligned}
v_{G B}^{1}\left[\lambda_{2}^{1}, \lambda_{2}^{2}\right]= & -x_{2}^{1}\left(a_{2}-\omega_{2}\right)^{2} \\
= & -\frac{1}{2} x_{2}^{1}\left[\gamma\left\{\left(P_{0,1}^{2,1}\right)^{2}+\left(P_{1,1}^{2,1}-1\right)^{2}\right\}\right. \\
& \left.+(1-\gamma)\left\{\left(P_{1,1}^{2,1}\right)^{2}+\left(P_{0,1}^{2,1}-1\right)^{2}\right\}\right]
\end{aligned}
$$

when he knows that the other advisor is of the bad type. With probability $\gamma$, the good advisor obtains the signal which is the same as the state of the world in each state of the world. Also, the good advisor knows that the other advisor sends the message 1 regardless of the signal. When the good advisor knows that the other advisor is also of the good type, the value function of the good advisor is 


$$
\begin{aligned}
v_{G G}^{1}\left[\lambda_{2}^{1}, \lambda_{2}^{2}\right]= & -x_{2}^{1}\left(a_{2}-\omega_{2}\right)^{2} \\
= & -\frac{1}{2} x_{2}^{1}\left[\gamma^{2}\left\{\left(P_{0,0}^{2,1}\right)^{2}+\left(P_{1,1}^{2,1}-1\right)^{2}\right\}\right. \\
& +\gamma(1-\gamma)\left\{\left(P_{0,1}^{2,1}\right)^{2}+\left(P_{1,0}^{2,1}\right)^{2}\right. \\
& \left.+\left(P_{1,0}^{2,1}-1\right)^{2}+\left(P_{0,1}^{2,1}-1\right)^{2}\right\} \\
& \left.+(1-\gamma)^{2}\left\{\left(P_{1,1}^{2,1}\right)^{2}+\left(P_{0,0}^{2,1}-1\right)^{2}\right\}\right] .
\end{aligned}
$$

With probability $\gamma^{2}$, both advisors obtain the signal which is the same as the state of the world in each state of the world. One advisor obtains the signal which is the same as the state of the world and the other advisor is misinformed with probability $\gamma(1-\gamma)$ in each state of the world.

Irrespective of his type and the type of the other advisor, the value function for an advisor is increasing with the updated belief of the decision maker that an advisor is good. If the message in the first period increases the updated belief of the decision maker that the advisor is good, it can also increase the value function in the second period.

\subsection{First Period}

In the first period, the payoff of the bad advisor is either

$$
y_{1}^{1} a_{1}+v_{B G}^{1}\left[\lambda_{2}^{1}\left(\lambda_{1}^{1}, m_{1}^{1}, \omega_{1}\right), \lambda_{2}^{2}\left(\lambda_{1}^{2}, m_{1}^{2}, \omega_{1}\right)\right]
$$

or

$$
y_{1}^{1} a_{1}+v_{B B}^{1}\left[\lambda_{2}^{1}\left(\lambda_{1}^{1}, m_{1}^{1}, \omega_{1}\right), \lambda_{2}^{2}\left(\lambda_{1}^{2}, m_{1}^{2}, \omega_{1}\right)\right]
$$

by following the type of the other advisor. The payoff of the good advisor is expressed as

$$
-x_{1}^{1}\left(a_{1}-\omega_{1}\right)^{2}+v_{G B}^{1}\left[\lambda_{2}^{1}\left(\lambda_{1}^{1}, m_{1}^{1}, \omega_{1}\right), \lambda_{2}^{2}\left(\lambda_{1}^{2}, m_{1}^{2}, \omega_{1}\right)\right]
$$

or

$$
-x_{1}^{1}\left(a_{1}-\omega_{1}\right)^{2}+v_{G G}^{1}\left[\lambda_{2}^{1}\left(\lambda_{1}^{1}, m_{1}^{1}, \omega_{1}\right), \lambda_{2}^{2}\left(\lambda_{1}^{2}, m_{1}^{2}, \omega_{1}\right)\right] .
$$

Suppose that good advisor sometimes tells a lie. The bad advisor also sometimes tells a lie, i.e. he does have a current payoff incentive to tell a lie if he observes the signal 0 and have a reputational incentive to tell a lie if 
he observes the signal 1 . If advisor $j$ is good, he sends the message 0 when his signal is 0 , and sends the message 1 with probability $z$ when the signal is 1 . If advisor $j$ is bad, he sends the message 1 with probability $\nu$ when his signal is 0 and sends the message 1 with probability $\rho$ when the signal is 1 . It is shown that the probability that good advisor sends the message 0 if his signal is 1 increases with the probability that bad advisor sends the message 1 if his signal is 1 . Since this model examines the case where the good advisor can distinguish his type from the bad type by sending the message the bad advisor is not biased towards, let us consider the case where the bad advisor sends the message 1 more often than good advisor, i.e. $\rho \geq z$. By sending the message 1, the likelihood that the advisor is bad increases.

By using Bayes' rule, the decision maker calculates the updated belief about the type of each advisor by using the message and the revealed state of the world. The updated belief of the decision maker that advisor $j$ is good, when the decision maker receives the message 1 and the real state of the world is revealed as 1 , is

$$
\lambda_{2}^{j}\left(\lambda_{1}^{j}, 1,1\right)=\frac{\lambda_{1}^{j} \gamma z}{\lambda_{1}^{j} \gamma z+\left(1-\lambda_{1}^{j}\right)\{\gamma \rho+(1-\gamma) \nu\}} .
$$

If the decision maker receives the message 1 from advisor $j$, he believes that the advisor $j$ is either of the good type or of the bad type. The real state of the world is revealed as 1 . The advisor $j$ obtains the signal 1 with probability $\gamma$. The decision maker believes that the advisor $j$, if advisor $j$ is of the good type, sends the message 1 with probability $\gamma z$. He also believes that the advisor $j$, if advisor $j$ is of the bad type, sends the message 1 with probability $\{\gamma \rho+(1-\gamma) \nu\}$. By using the same method, the updated belief of the decision maker for each message and each state of the world is

$$
\begin{gathered}
\lambda_{2}^{j}\left(\lambda_{1}^{j}, 0,1\right)=\frac{\lambda_{1}^{j}(1-\gamma z)}{\lambda_{1}^{j}(1-\gamma z)+\left(1-\lambda_{1}^{j}\right)\{1-\gamma \rho-(1-\gamma) \nu\}}, \\
\lambda_{2}^{j}\left(\lambda_{1}^{j}, 0,0\right)=\frac{\lambda_{1}^{j}\{1-(1-\gamma) z\}}{\lambda_{1}^{j}\{1-(1-\gamma) z\}+\left(1-\lambda_{1}^{j}\right)\{1-(1-\gamma) \rho-\gamma \nu\}}
\end{gathered}
$$

and

$$
\lambda_{2}^{j}\left(\lambda_{1}^{j}, 1,0\right)=\frac{\lambda_{1}^{j}(1-\gamma) z}{\lambda_{1}^{j}(1-\gamma) z+\left(1-\lambda_{1}^{j}\right)\{(1-\gamma) \rho+\gamma \nu\}} .
$$


Proposition 1 Regardless of the state of the world in the first period, advisor $j$ has reputational incentive to announce 0 because

$$
\lambda_{2}^{j}\left(\lambda_{1}^{j}, 0,0\right)>\lambda_{1}^{j}>\lambda_{2}^{j}\left(\lambda_{1}^{j}, 1,0\right)
$$

and

$$
\lambda_{2}^{j}\left(\lambda_{1}^{j}, 0,1\right)>\lambda_{1}^{j}>\lambda_{2}^{j}\left(\lambda_{1}^{j}, 1,1\right)
$$

Under the condition that bad advisor sends the message 1 more often than good advisor, each type of advisor has reputational incentive to send the message 0 in the first period. Even if real state of the world in the first period is revealed as 1 , sending the message 0 is the way to increase the reputation. It is because each advisor knows that there is imperfect signal regarding the state of the world and to send the message 0 is the way to separate his type from the bad type. In the example, each type of market expert has reputational incentive to suggest the defensive pricing policy to the manager of the firm regardless of his signal. It is because the likelihood that the expert is of the aggressive type decreases when the expert suggests the defensive pricing strategy. Proposition 1 will help me consider both good and bad reputation effects.

In order to consider the payoff in the first period, the belief of the decision maker that the state of the world in the first period is 1 given each pair of messages is calculated. If the decision maker receives the message 0 from both advisors, the probability that the state of the world is 1 is

$$
P_{0,0}^{1,1}=\frac{Q_{0,0}^{1,1}}{Q_{0,0}^{1,0}+Q_{0,0}^{1,1}}
$$

where $Q_{m_{1}^{1}, m_{1}^{2}}^{1, l}$ represents the conditional probability that the message of the first advisor is $m_{1}^{1}$ and the message of the second advisor is $m_{1}^{2}$ given that the state of the world in the first period is $l$, and

$$
\begin{aligned}
Q_{0,0}^{1,0}= & {\left[\lambda_{1}^{1}\{1-(1-\gamma) z\}+\left(1-\lambda_{1}^{1}\right)\{1-(1-\gamma) \rho-\gamma \nu\}\right] \times } \\
& {\left[\lambda_{1}^{2}\{1-(1-\gamma) z\}+\left(1-\lambda_{1}^{2}\right)\{1-(1-\gamma) \rho-\gamma \nu\}\right] }
\end{aligned}
$$

and

$$
\begin{aligned}
Q_{0,0}^{1,1}= & {\left[\lambda_{1}^{1}(1-\gamma z)+\left(1-\lambda_{1}^{1}\right)\{1-\gamma \rho-(1-\gamma) \nu\}\right] \times } \\
& {\left[\lambda_{1}^{2}(1-\gamma z)+\left(1-\lambda_{1}^{2}\right)\{1-\gamma \rho-(1-\gamma) \nu\}\right] }
\end{aligned}
$$


The probability that both advisors send the message 0 is calculated by considering the 32 possible cases. In each state of the world, the good advisor sends the message 0 if his signal is 0 , and sends the message 0 with probability $1-z$ if the signal is 1 . The bad advisor sends the message 0 with probability $1-\nu$ if his signal is 0 , and sends the message 0 with probability $1-\rho$ if the signal is 1 . The decision maker believes that each advisor may be either of the good or of the bad type. The decision maker chooses action $P_{0,0}^{1,1}$ when he receives the message 0 from both advisors in the first period.

The probability that the state of the world in the first period is 1 when the decision maker receives $m_{1}^{1}=0$ and $m_{1}^{2}=1$ is

$$
P_{0,1}^{1,1}=\frac{Q_{0,1}^{1,1}}{Q_{0,1}^{1,0}+Q_{0,1}^{1,1}}
$$

where

$$
\begin{aligned}
Q_{0,1}^{1,0}= & {\left[\lambda_{1}^{1}\{1-(1-\gamma) z\}+\left(1-\lambda_{1}^{1}\right)\{1-(1-\gamma) \rho-\gamma \nu\}\right] \times } \\
& {\left[\lambda_{1}^{2}(1-\gamma) z+\left(1-\lambda_{1}^{2}\right)\{(1-\gamma) \rho+\gamma \nu\}\right] }
\end{aligned}
$$

and

$$
\begin{aligned}
Q_{0,1}^{1,1}= & {\left[\lambda_{1}^{1}(1-\gamma z)+\left(1-\lambda_{1}^{1}\right)\{1-\gamma \rho-(1-\gamma) \nu\}\right] \times } \\
& {\left[\lambda_{1}^{2} \gamma z+\left(1-\lambda_{1}^{2}\right)\{\gamma \rho+(1-\gamma) \nu\}\right] . }
\end{aligned}
$$

Since the good advisor who observes the signal 0 never sends the message 1 , the decision maker believes that the second advisor is either good advisor who observes the signal 1 or bad advisor in each state of the world. The decision maker chooses action $P_{0,1}^{1,1}$ when he receives the message 0 from the first advisor and the message 1 from the second advisor in the first period.

The probability that the state of the world in the first period is 1 if the decision maker receives $m_{1}^{1}=1$ and $m_{1}^{2}=0$ is

$$
P_{1,0}^{1,1}=\frac{Q_{1,0}^{1,1}}{Q_{1,0}^{1,0}+Q_{1,0}^{1,1}}
$$

where

$$
\begin{aligned}
Q_{1,0}^{1,0}= & {\left[\lambda_{1}^{1}(1-\gamma) z+\left(1-\lambda_{1}^{1}\right)\{(1-\gamma) \rho+\gamma \nu\}\right] \times } \\
& {\left[\lambda_{1}^{2}\{1-(1-\gamma) z\}+\left(1-\lambda_{1}^{2}\right)\{1-(1-\gamma) \rho-\gamma \nu\}\right] }
\end{aligned}
$$


and

$$
\begin{aligned}
Q_{1,0}^{1,1}= & {\left[\lambda_{1}^{1} \gamma z+\left(1-\lambda_{1}^{1}\right)\{\gamma \rho+(1-\gamma) \nu\}\right] \times } \\
& {\left[\lambda_{1}^{2}(1-\gamma z)+\left(1-\lambda_{1}^{2}\right)\{1-\gamma \rho-(1-\gamma) \nu\}\right] . }
\end{aligned}
$$

When the decision maker receives the message 1 from the first advisor and the message 0 from the second advisor, he chooses action $P_{1,0}^{1,1}$ in the first period.

If both advisors send the message 1 , the probability that the state of the world in the first period is 1 is

$$
P_{1,1}^{1,1}=\frac{Q_{1,1}^{1,1}}{Q_{1,1}^{1,0}+Q_{1,1}^{1,1}}
$$

where

$$
\begin{aligned}
Q_{1,1}^{1,0}= & {\left[\lambda_{1}^{1}(1-\gamma) z+\left(1-\lambda_{1}^{1}\right)\{(1-\gamma) \rho+\gamma \nu\}\right] \times } \\
& {\left[\lambda_{1}^{2}(1-\gamma) z+\left(1-\lambda_{1}^{2}\right)\{(1-\gamma) \rho+\gamma \nu\}\right] }
\end{aligned}
$$

and

$$
\begin{aligned}
Q_{1,1}^{1,1}= & {\left[\lambda_{1}^{1} \gamma z+\left(1-\lambda_{1}^{1}\right)\{\gamma \rho+(1-\gamma) \nu\}\right] \times } \\
& {\left[\lambda_{1}^{2} \gamma z+\left(1-\lambda_{1}^{2}\right)\{\gamma \rho+(1-\gamma) \nu\}\right] }
\end{aligned}
$$

and the decision maker chooses action $P_{1,1}^{1,1}$ in the first period. The decision maker believes that the advisor who sends the message 1 is either of the good or of the bad type. Since good advisor who observes the signal 0 never sends the message 1, the decision maker infers that the good advisor who sends the message 1 observes the signal 1 . He also infers that the bad advisor who observes the signal either 0 or 1 sends the message 1 with some probability in each state of the world.

Under the conditions that each advisor has imperfect information regarding the state of the world and has perfect information regarding the type of the other advisor, I first examine the existence of good reputation effect. Let us consider the case where the first advisor who is of the bad type observes the signal 0 and knows that the second advisor is good. The bad advisor believes that the second advisor sends the message 0 with probability 1 if his signal is 0 , or sends the message 1 with probability $z$ if his signal is 1 . Since each advisor obtains the correct signal regarding the state 
of the world with probability $\gamma$, the belief of the first advisor that the signal of the second advisor is the same as that of the first advisor is $\frac{1}{2}$.

The bad advisor's total utility of telling the truth $\left(m_{1}^{1}=0\right)$ when he observes the signal 0 is

$$
\begin{aligned}
& \frac{1}{2}\left[y_{1}^{1}\left\{2 P_{0,0}^{1,1}+z\left(P_{0,1}^{1,1}-P_{0,0}^{1,1}\right)\right\}\right. \\
& +\sum_{\epsilon=0}^{1} \sum_{\zeta=0}^{1} v_{B G}^{1}\left[R_{\epsilon} \lambda_{2}^{1}\left(\lambda_{1}^{1}, 0, \epsilon\right)\right. \\
& \left.\left.\frac{1}{2}\left\{R_{\epsilon} \lambda_{2}^{2}\left(\lambda_{1}^{2}, 0, \epsilon\right)+\left(1-R_{\epsilon}\right) z_{\zeta} \lambda_{2}^{2}\left(\lambda_{1}^{2}, \zeta, \epsilon\right)\right\}\right]\right]
\end{aligned}
$$

where $R_{0}=\gamma, R_{1}=1-\gamma, z_{0}=1-z$ and $z_{1}=z$. The bad advisor who sends the message 0 believes that the other advisor sends the message 0 if the signal of the other advisor is the same as his own signal. He also believes that the other advisor sends the message 0 with probability $1-z$ if the signal of the other advisor is different from his own signal. When the real state of the world is revealed to be 1, i.e. when the bad advisor is misinformed, he needs to consider the cases where the other advisor obtains the correct signal or is also misinformed. Similarly, the bad advisor needs to consider the case where the other advisor obtains the correct signal or is misinformed when the real state of the world is revealed to be 0 , i.e. when the bad advisor obtains the correct signal. The total utility to the bad advisor who observes the signal 0 when he tells a lie $\left(m_{1}^{1}=1\right)$ is

$$
\begin{aligned}
& \frac{1}{2}\left[y_{1}^{1}\left\{2 P_{1,0}^{1,1}+z\left(P_{1,1}^{1,1}-P_{1,0}^{1,1}\right)\right\}\right. \\
& +\sum_{\epsilon=0}^{1} \sum_{\zeta=0}^{1} v_{B G}^{1}\left[R_{\epsilon} \lambda_{2}^{1}\left(\lambda_{1}^{1}, 1, \epsilon\right),\right. \\
& \left.\left.\frac{1}{2}\left\{R_{\epsilon} \lambda_{2}^{2}\left(\lambda_{1}^{2}, 0, \epsilon\right)+\left(1-R_{\epsilon}\right) z_{\zeta} \lambda_{2}^{2}\left(\lambda_{1}^{2}, \zeta, \epsilon\right)\right\}\right]\right] .
\end{aligned}
$$

If the bad advisor only considers his payoff in the first period, i.e. $y_{1}^{1}=1$ and $y_{2}^{1}=0$, then he will send the message 1 after observing the signal 0 . Even if the bad advisor weights the two periods equally, i.e. $y_{1}^{1}=\frac{1}{2}=y_{2}^{1}$, he will prefer telling a lie. If the bad advisor only considers his second period payoff, i.e. $y_{1}^{1}=0$ and $y_{2}^{1}=1$, the payoff when he tells the truth is greater 
than the payoff when he tells a lie. Truth telling is possible if $y_{2}^{1}$ is sufficiently large. The critical value of $y_{1}^{1}$ which guarantees the existence of the good reputation effect is calculated as a function of parameters.

Proposition 2 There is good reputation effect for the advisor who observes the signal 0 and knows that the other advisor is good if he considers his second period as sufficiently more important (see Appendix A).

If the bad advisor who knows that the other advisor is good strongly considers his reputation, then after observing the signal 0 in the first period he sends the message 0 . This reputational concern that makes the bad advisor tell the truth is referred to the good reputation effect. In the example of the market expert and the manager of the firm, when the aggressive expert concludes that the defensive pricing policy is needed, the strong reputational concern of the expert not to be perceived as an aggressive expert makes him suggest the defensive pricing strategy to the manager of the firm even if he has loss in the current payoff. This result holds when the aggressive expert (bad expert) knows that the other expert is good expert.

Let us consider a numerical example to examine the relationship between the probability that the bad advisor sends the message truthfully if his signal is $0(\nu)$ and the belief of the decision maker about the type of each advisor, $\lambda_{1}^{1}$ and $\lambda_{1}^{2}$. It is supposed that the first advisor is of the bad type and the second advisor is of the good type. If $\gamma=\frac{2}{3}, y_{1}^{1}=\frac{1}{10}$ and $y_{2}^{1}=\frac{9}{10}$, i.e. if the bad advisor considers his second period as more important, the probability that the bad advisor sends the message truthfully if his signal is $0(1-\nu)$ is a function of $\lambda_{1}^{1}, \lambda_{1}^{2}, \rho$ and $z$. In Morris' paper, when the prior belief of the decision maker regarding the type of an advisor is either very low or very high, $\nu$ is high, i.e., the probability that the advisor tells a lie after observing the signal 0 is high. However, this paper shows that the belief of the decision maker about the type of the good advisor (the type of the other advisor) also can change the probability that the bad advisor tells a lie. If $\lambda_{1}^{1}=\frac{1}{2}=\lambda_{1}^{2}$, the value of $\nu$ lies between 0 and 1 . This in turn guarantees that the bad advisor tells the truth with a non-zero probability of $1-\nu$. Given $\lambda_{1}^{1}=\frac{1}{2}$, the probability that the bad advisor tells the truth increases with $\lambda_{1}^{2}$, i.e. $\nu$ decreases with $\lambda_{1}^{2}$. Especially if the prior belief of the decision maker about the good advisor $\left(\lambda_{1}^{2}\right)$ approaches $1, \nu$ is at its lowest value. The probability that the bad advisor tells the truth in the first period is very high if the decision maker believes the other advisor to be good with a 
very high probability given that the prior belief of the decision maker that the bad advisor is good is $\frac{1}{2}$.

If the prior belief of the decision maker about the bad advisor is very high, i.e. if $\lambda_{1}^{1}$ is very high, the bad advisor becomes more likely to tell a lie if the prior belief of the decision maker about the type of the good advisor $\left(\lambda_{1}^{2}\right)$ approaches 0 . The bad advisor becomes more likely to tell the truth if $\lambda_{1}^{2}$ approaches 1 . It is because the reputation of the bad advisor cannot decrease a lot if the prior belief of the decision maker about him $\left(\lambda_{1}^{1}\right)$ is very high. The bad advisor will have a greater fear of losing his reputation if the belief of the decision maker about the type of the good advisor $\left(\lambda_{1}^{2}\right)$ is very high.

If the prior belief of the decision maker about the bad advisor, $\lambda_{1}^{1}$, is very low, the incentive to tell the truth increases when $\lambda_{1}^{2}$ is also very low. The incentive to tell a lie increases if $\lambda_{1}^{2}$ increases given very low $\lambda_{1}^{1}$. The bad advisor knows that it is very hard to increase his reputation if the prior belief of the decision maker about his type is very low. However, if the belief of the decision maker about type of both advisors is very low, it is relatively easy for the bad advisor to increase his reputation. In each case, the presence of the other advisor (especially the belief of the decision maker about the type of the other advisor) plays an important role in determining the choice of the message of one advisor.

Next is the case where the first advisor who is of bad type observes the signal 0 , and knows that the second advisor is also bad. Under the belief that the bad advisor sends the message 1 with probability $\nu$ if the signal is 0 , or sends the message 1 with probability $\rho$ if the signal is 1 , the first advisor compares the total utility when he tells the truth with that when he tells a lie. There is good reputation effect for the advisor who knows the other advisor is also bad if he puts a relatively greater weight on the second period payoff (see Appendix B). It is shown that the area which guarantees the existence of good reputation effect is bigger when the bad advisor faces a good advisor rather than a bad advisor. This is because the good advisor sends the message 0 more often than the bad advisor. The strong reputational concern not to be perceived as the bad advisor makes the bad advisor send the message 0 more easily when he knows that the other advisor is of the good type rather than of the bad type.

In order to examine the existence of bad reputation effect, let us consider the case where the first advisor who is of the good type observes the signal 
1 and meets the bad advisor. The good advisor knows that the bad advisor sends the message 1 with probability $\nu$ if the signal is 0 , or sends the message 1 with probability $\rho$ if the signal is 1 . The good advisor compares the total utility of sending message truthfully $\left(m_{1}^{1}=1\right)$ with the total utility of telling a lie $\left(m_{1}^{1}=0\right)$.

The total utility to the good advisor from sending message truthfully $\left(m_{1}^{1}=1\right)$ is

$$
\begin{aligned}
& -\frac{1}{2} x_{1}^{1} \sum_{\epsilon=0}^{1} \sum_{\zeta=0}^{1}\left[\frac { 1 } { 2 } \left[\rho_{\epsilon}\left\{\gamma^{2}\left(P_{1, \epsilon}^{1,1}-1\right)^{2}+(1-\gamma)^{2}\left(P_{1, \epsilon}^{1,1}\right)^{2}\right\}\right.\right. \\
& \left.+\gamma(1-\gamma) \nu_{\epsilon}\left\{\left(P_{1, \epsilon}^{1,1}-1\right)^{2}+\left(P_{1, \epsilon}^{1,1}\right)^{2}\right\}\right] \\
& +v_{G B}^{1}\left[\left(1-R_{\epsilon}\right) \lambda_{2}^{1}\left(\lambda_{1}^{1}, 1, \epsilon\right)\right. \\
& \left.\left.\frac{1}{2}\left\{\left(1-R_{\epsilon}\right) \rho_{\zeta} \lambda_{2}^{2}\left(\lambda_{1}^{2}, \zeta, \epsilon\right)+R_{\epsilon} \nu_{\zeta} \lambda_{2}^{2}\left(\lambda_{1}^{2}, \zeta, \epsilon\right)\right\}\right]\right]
\end{aligned}
$$

where $R_{0}=\gamma, R_{1}=1-\gamma, \rho_{0}=1-\rho, \rho_{1}=\rho, \nu_{0}=1-\nu$ and $\nu_{1}=\nu$. The state of the world is equally likely and the belief of the good advisor that the other advisor obtains the same signal is $\frac{1}{2}$. In the case where the good advisor is misinformed, i.e. the real state of the world is revealed as 0 , the good advisor needs to consider the case where the other advisor is also misinformed or obtains the correct signal. The good advisor also needs to consider the case where the other advisor obtains the correct signal or is misinformed when he obtains the correct signal, i.e. when the real state of the world is 1 . The total utility to the good advisor from telling a lie $\left(m_{1}^{1}=0\right)$ is

$$
\begin{aligned}
& -\frac{1}{2} x_{1}^{1} \sum_{\epsilon=0}^{1} \sum_{\zeta=0}^{1}\left[\frac { 1 } { 2 } \left[\rho_{\epsilon}\left\{\gamma^{2}\left(P_{0, \epsilon}^{1,1}-1\right)^{2}+(1-\gamma)^{2}\left(P_{0, \epsilon}^{1,1}\right)^{2}\right\}\right.\right. \\
& \left.+\gamma(1-\gamma) \nu_{\epsilon}\left\{\left(P_{0, \epsilon}^{1,1}-1\right)^{2}+\left(P_{0, \epsilon}^{1,1}\right)^{2}\right\}\right] \\
& +v_{G B}^{1}\left[\left(1-R_{\epsilon}\right) \lambda_{2}^{1}\left(\lambda_{1}^{1}, 0, \epsilon\right)\right. \\
& \left.\left.\frac{1}{2}\left\{\left(1-R_{\epsilon}\right) \rho_{\zeta} \lambda_{2}^{2}\left(\lambda_{1}^{2}, \zeta, \epsilon\right)+R_{\epsilon} \nu_{\zeta} \lambda_{2}^{2}\left(\lambda_{1}^{2}, \zeta, \epsilon\right)\right\}\right]\right]
\end{aligned}
$$

If the good advisor only cares about the first period payoff, i.e. $x_{1}^{1}=1$ and $x_{2}^{1}=0$, then the good advisor will send message truthfully $\left(m_{1}^{1}=1\right)$ after observing the signal 1 . If the good advisor only cares about the second period, i.e. $x_{1}^{1}=0$ and $x_{2}^{1}=1$, then he tells a lie $\left(m_{1}^{1}=0\right)$ to increase 
his reputation. The truth telling is possible if $x_{1}^{1}$ is sufficiently large. The critical value of $x_{1}^{1}$ which guarantees the existence of the bad reputation effect is calculated as the function of the parameters. Below the critical point of $x_{1}^{1}$, there is equilibrium where the good advisor sometimes tells a lie.

Proposition 3 There is bad reputation effect for the advisor who observes the signal 1 and knows that the other advisor is bad if he considers his second period as sufficiently more important (see Appendix C).

The strong reputational concern of the good advisor who knows that the other advisor is bad makes him send the message 0 in the first period after observing the signal 1 . Consider the example where the good market expert who knows that the other expert is of the aggressive type concludes that aggressive pricing policy is needed. If the good expert strongly does not want to be perceived as the aggressive expert, he suggests the defensive pricing strategy to the manager of the firm. It is because he wants the decision maker to believe what he will suggest next time.

If $y_{1}^{2}=\frac{1}{2}=y_{2}^{2}$, i.e. if the second advisor weights the two periods equally, it is shown that the bad advisor always sends the message 1 in the first period. The good advisor who observes the signal 1 sends the message 0 if he puts the greater weight on the second period (if $x_{1}^{1}<0.2923$ for $\lambda_{1}^{1}=\frac{1}{2}=\lambda_{1}^{2}$ and $\gamma=\frac{2}{3}$ ). Compared to the example in Morris' paper, the existence of the other advisor reinforces the bad reputation effect.

If the bad advisor sometimes sends the message 0 (this happens for $y_{1}^{2}=\frac{1}{10}, y_{2}^{2}=\frac{9}{10}$ and $\left.\lambda_{1}^{1}=\frac{1}{2}=\lambda_{1}^{2}\right)$, the area which guarantees the existence of the bad reputation effect is smaller than the area which guarantees the existence of the bad reputation effect when the bad advisor always sends the message 1. It is because the area which guarantees the existence of the bad reputation effect (the critical value) increases with the probability that bad advisor sends the message 1 if his signal is $1(\rho)$. The good advisor has greater incentive to tell a lie (i.e. he has greater incentive to send the message 0 after observing the signal 1 ) in order to increase his reputation if the other bad advisor always sends the message 1 .

If the bad advisor always sends the message 0 and the prior belief of the decision maker about the type of each advisor is $\frac{1}{2}$, i.e. if $y_{1}^{2}=0$, 
$y_{2}^{2}=1$ and $\lambda_{1}^{1}=\frac{1}{2}=\lambda_{1}^{2}$, the good advisor also sends the message 0 if he considers his second period as sufficiently more important (if $x_{1}^{1}<0.2692$ ). This is pooling equilibrium in the first period. In this case, if the real state of the world is revealed as 1 in the first period, the decision maker loses all information regarding the state of the world from having an additional advisor.

When the good advisor observes the signal 1 and knows that the other advisor is also good (and will send the message 0 if his signal is 0 , or the message 1 with probability $z$ if the signal is 1 ), there is bad reputation effect for the advisor if he puts greater weight on the second period payoff (see Appendix D). The area which guarantees the existence of bad reputation effect is bigger when the good advisor faces a bad rather than a good advisor. Since the bad advisor sends the message 1 more often than the good advisor, the good advisor has greater incentive to tell a lie when he meets the other bad advisor in order to separate himself from the bad type.

\section{$3 \quad$ Welfare Effect}

The decision maker tries to obtain additional information with an additional advisor. However, bad reputation effect arises if an advisor has strong reputational concern to be perceived as a good advisor. To examine the welfare of the decision maker, I compare the payoff of the decision maker with one advisor and with two advisors. For simplicity, it is assumed that the decision maker believes that an advisor is good with probability $\frac{1}{2}$ before the first period starts.

In order to calculate the expected payoff of the decision maker when he has a single advisor, the action of the decision maker given each message is calculated in each period. Since the second period is the last period, the good advisor sends the message which is the same as his signal and the bad

advisor always sends the message 1. From Morris' paper, the probability that the state of the world is 1 in the second period given each message is

$$
P_{0}^{2,1}=1-\gamma
$$

and

$$
P_{1}^{2,1}=\frac{1-\lambda_{2}^{1}+\lambda_{2}^{1} \gamma}{2-\lambda_{2}^{1}}
$$


where $P_{m_{i}^{1}}^{i, 1}$ represents the probability that the state of the world in period $i$ is 1 given the message of one advisor $m_{i}^{1}$.

In the first period, the good advisor sends the message 0 if his signal is 0 in the first period and sends the message 1 with probability $z$ if the signal is 1 . The bad advisor sends the message 1 with probability $\rho$ if his signal is 1 and sends the message 1 with probability $\nu$ if his signal is 0 . The probability that state of the world in the first period is 1 given the message of an advisor is

$$
P_{0}^{1,1}=\frac{\lambda_{1}^{1}(1-\gamma z)+\left(1-\lambda_{1}^{1}\right)\{1-\gamma \rho-(1-\gamma) \nu\}}{\lambda_{1}^{1}(2-z)+\left(1-\lambda_{1}^{1}\right)(2-\rho-\nu)}
$$

and

$$
P_{1}^{1,1}=\frac{\lambda_{1}^{1} \gamma z+\left(1-\lambda_{1}^{1}\right)\{\gamma \rho+(1-\gamma) \nu\}}{\lambda_{1}^{1} z+\left(1-\lambda_{1}^{1}\right)(\rho+\nu)}
$$

I first separate each period payoff of the decision maker when he has two advisors or has one advisor, and then compare the total payoff of the decision maker. If each type of the advisor considers his second period as sufficiently more important, each type of advisor sends the message 0 in the first period regardless of his signal (for example, $y_{2}^{2}=1$ and $x_{2}^{1}=1$ ). The payoff of the decision maker when he has two advisors is

$$
-\frac{1}{2}\left\{\left(P_{0,0}^{1,1}\right)^{2}+\left(P_{0,0}^{1,1}-1\right)^{2}\right\}
$$

and that when he has a single advisor is

$$
-\frac{1}{2}\left\{\left(P_{0}^{1,1}\right)^{2}+\left(P_{0}^{1,1}-1\right)^{2}\right\}
$$

The probability that the state of the world in the first period is 0 given that both advisors send the message 0 is greater than the probability that the state of the world in the first period is 0 given that one advisor sends the message 0, i.e. $P_{0,0}^{1,0}>P_{0}^{1,0}$ or $P_{0,0}^{1,1}<P_{0}^{1,1}$. If the state of the world is $0,\left(P_{0,0}^{1,1}\right)^{2}$ is less than $\left(P_{0}^{1,1}\right)^{2}$. If the state of the world is $1,\left(P_{0,0}^{1,1}-1\right)^{2}$ is greater than $\left(P_{0}^{1,1}-1\right)^{2}$. Since the welfare loss of having two advisors if the state of the world is 1 is a lot greater than that of having a single advisor if the state of the world in the first period is 1 , compared with the welfare gain of having two advisors if the state of the world is 0 , it is better for the decision maker to have a single advisor in the first period. 
In the second period, the expected payoff of the decision maker from having two advisors is calculated by considering the cases when both advisors are good, one advisor is good and the other advisor is bad, and both advisors are bad. The expected payoff of the decision maker when he has a single advisor in the second period is also calculated by considering the case when the advisor is good or the advisor is bad (Appendix E). In the second period, the welfare loss from obtaining the wrong signal if the decision maker has two advisors is a lot greater than that if the decision maker has a single advisor, compared with the welfare gain which is from obtaining the correct signal. It is shown that having one good advisor is always better than having two advisors regardless of types of both advisors. To have at least one good advisor if the decision maker has two advisors is better than to have a single bad advisor. However, it is better for the decision maker to have a single bad advisor than two bad advisors. Since it is assumed that each advisor is good with probability $\frac{1}{2}$, the comparison of the total payoff of the decision maker between two advisors case and single advisor case leads to the following proposition.

Proposition 4 Under the condition that each type of the advisor considers his second period as sufficiently more important, the decision maker cannot benefit of taking advice from an additional advisor.

If the strong reputational concern makes each type of the advisor send the message 0 in the first period, the decision maker is better off having a single advisor rather than two advisors. If the bad advisor always sends the message 1 in the first period (for example, $y_{1}^{2}=\frac{1}{2}=y_{2}^{2}$ ), and if the good advisor considers his second period as sufficiently more important, it is better for the decision maker to have two advisors. If each type of the advisor sometimes sends the message 1, the decision maker can benefit from taking advice from an additional advisor. Except the case where each type of advisor considers his second period as sufficiently more important, it is better for the decision maker to have two advisors.

\section{Conclusion}

In this paper, I first characterize the conditions for the existence of both good and bad reputation effects when each advisor knows the type of the 
other advisor, and the advisors send the message simultaneously. In any informative equilibrium, regardless of the signal both advisors have reputational incentive to send the message the bad advisor is not biased towards. By comparing the total payoff of telling the truth and that of telling a lie, I show that bad advisor sometimes tells the truth to increase his reputation and show that strong reputational concern makes the good advisor sometimes tell a lie regardless of the type of the other advisor. Moreover, bad (good) reputation effect is more likely to emerge when the good (bad) advisor knows that the other advisor is bad (good) rather than good (bad). I then examine whether the decision maker is better off if he obtains information from an additional advisor. The expected payoff of the decision maker is lower with two advisors than with only one advisor if each type of the advisor considers his second period as sufficiently more important.

I can extend this model to analyze the case where each advisor has imperfect information regarding the type of the other advisor. I have shown in this paper that the presence of the other advisor can affect the message of an advisor when each advisor knows the type of the other advisor. If each advisor has imperfect information about the type of the other advisor, the strategic choice of an advisor may be changed, which may lead to different results about both good and bad reputation effects. Also, I believe that the results may be different if this game is repeated finitely. Especially, when the good advisor observes the high signal continuously, I believe that the possibility of the existence of bad reputation effect might increase because the reputational concern may increase with the period. The case where advisors each of whom knows the type of the other advisor send the messages sequentially to the decision maker may lead to the different results. When the decision maker asks for advice from an additional advisor, he informs the second advisor of the message sent by the first advisor. The second advisor adjusts his message by following the message of the first advisor. By examining the total payoff of the decision maker, I may determine whether simultaneous advice or sequential advice is preferred by the decision maker.

\section{Appendix A}

The value function of the first advisor, if he is of the bad type, is calculated using the probability that the state of the world is 1 given his message is 1 . The value function for the bad advisor is

$$
v_{B G}^{1}\left[\lambda_{2}^{1}, \lambda_{2}^{2}\right]=y_{2}^{1} a_{2}=\frac{1}{2} y_{2}^{1}\left(P_{1,0}^{2,1}+P_{1,1}^{2,1}\right)
$$


when the bad advisor meets the good advisor.

There is good reputation effect of the advisor who observes the signal 0 and meets the other advisor who is of the good type if

$$
y_{1}^{1}\left(-\alpha_{1}\right)+\beta_{1} y_{2}^{1}+\eta_{1} y_{2}^{1}>0
$$

where $y_{1}^{1}+y_{2}^{1}=1$

$$
\begin{aligned}
& \text { and }-\alpha_{1}=\sum_{\epsilon=0}^{1}(-1)^{\epsilon}\left\{\left(P_{\epsilon, 0}^{1,1}\right)\left(1-\frac{1}{2} z\right)+z\left(P_{\epsilon, 1}^{1,1}\right)\right\}<0 \\
& \beta_{1}=\frac{1}{4}\left\{\sum_{\epsilon=0}^{1}(-1)^{\epsilon} \frac{1-\gamma-\lambda_{2}^{1}\left(\lambda_{1}^{1}, \epsilon, 1\right)(1-\gamma)^{2}}{1-\lambda_{2}^{1}\left(\lambda_{1}^{1}, \epsilon, 1\right)\left(1-2 \gamma+2 \gamma^{2}\right)}+f_{1}\left(\lambda_{1}^{1}, \lambda_{1}^{2}, \gamma, z, \rho, \nu\right)\right\}>0 \text { and } \\
& \eta_{1}=\frac{1}{4}\left\{\sum_{\epsilon=0}^{1}(-1)^{\epsilon} \frac{1-\gamma-\lambda_{2}^{1}\left(\lambda_{1}^{1}, \epsilon, 0\right)(1-\gamma)^{2}}{1-\lambda_{2}^{1}\left(\lambda_{1}^{1}, \epsilon, 0\right)\left(1-2 \gamma+2 \gamma^{2}\right)}+f_{2}\left(\lambda_{1}^{1}, \lambda_{1}^{2}, \gamma, z, \rho, \nu\right)\right\}>0 .
\end{aligned}
$$

Here, $-\alpha_{1}$ explains the difference between the first advisor's first period payoff when he tells the truth and the payoff when he tells a lie. The bad advisor believes that the other advisor obtains the signal 0 with probability $\frac{1}{2}$. He also believes that the good advisor who observes the signal 0 sends the message 0 if his signal is 0 and sends the message 0 with probability $1-z$ if the signal is 1 , i.e. with probability $\frac{1}{2}(1-z)$ or $\frac{1}{2}$, the second advisor sends the message 0 .

$\beta_{1}$ shows the difference between the first advisor's second period payoff which is determined by the value function of the bad advisor when he tells the truth and the payoff when he tells a lie in the case where the real state of the world is revealed as 1 . If the first advisor believes that the second advisor observes the signal 0, i.e. the first advisor believes that the second advisor's signal is the same as his signal, he knows that the updated belief of the decision maker about the type of the second advisor is $\frac{1}{2}(1-\gamma) \lambda_{2}^{2}\left(\lambda_{1}^{2}, 0,1\right)$. It is because the second advisor is misinformed when the real state of the world is 1 . In the case as the first advisor believes that the second advisor observes the signal 1 , the updated belief of the decision maker that the second advisor is good is $\frac{1}{2} \gamma\left\{z \lambda_{2}^{2}\left(\lambda_{1}^{2}, 1,1\right)+(1-z) \lambda_{2}^{2}\left(\lambda_{1}^{2}, 0,1\right)\right\}$ since the good advisor who observes the signal 1 sends the message 0 with probability $1-z$.

$\eta_{1}$ shows the difference of the first advisor's second period payoff between telling the truth and telling a lie in the case where the real state of the world is revealed as 0 . Since the expression of $\beta_{1}$ and $\eta_{1}$ is so complicated, I use 
the functional expression $\left.f_{a}\left(\lambda_{1}^{1}, \lambda_{1}^{2}, \gamma, z, \rho, \nu\right)\right\}$ for $a=1$ or 2 to show the rest part of the difference between telling the truth and telling a lie when the state of the world is revealed as 1 or 0 respectively .

If the bad advisor who meets the other good advisor considers his second period as sufficiently more important, he sends the message he is not biased towards, i.e. if

$$
y_{1}^{1}<\frac{\beta_{1}+\eta_{1}}{\alpha_{1}+\beta_{1}+\eta_{1}}=F_{B}\left(\lambda_{1}^{1}, \lambda_{1}^{2}, \gamma, z, \rho, \nu\right)<\frac{1}{2},
$$

the bad advisor who observes the signal 0 sends the message 0 .

\section{Appendix B}

The expressions in Appendix B are very similar as those in Appendix A. The value function of the bad advisor, if the first advisor is of the bad type, is

$$
v_{B B}^{1}\left[\lambda_{2}^{1}, \lambda_{2}^{2}\right]=y_{2}^{1} a_{2}=y_{2}^{1} P_{1,1}^{2,1}
$$

when the bad advisor meets the bad advisor. If the bad advisor who meets the other bad advisor considers his second period as sufficiently more important, after observing the signal 0 he sends the message 0 .

I compare the area which guarantees the existence of the bad reputation effect when the bad advisor meets good advisor with the area which guarantees the existence of the bad reputation effect when the bad advisor meets the other bad advisor and find that the good reputation effect occurs more easily when the bad advisor meets the other good advisor.

\section{Appendix C}

If the first advisor is of the good type, the value function for the good advisor is

$$
\begin{aligned}
v_{G B}^{1}\left[\lambda_{2}^{1}, \lambda_{2}^{2}\right]= & -x_{2}^{1}\left(a_{2}-\omega_{2}\right)^{2} \\
= & -\frac{1}{2} x_{2}^{1}\left[\gamma\left(P_{0,1}^{2,1}\right)^{2}+(1-\gamma)\left(P_{1,1}^{2,1}\right)^{2}\right. \\
& \left.+\gamma\left(P_{1,1}^{2,1}-1\right)^{2}+(1-\gamma)\left(P_{0,1}^{2,1}-1\right)^{2}\right]
\end{aligned}
$$

when he knows that the second advisor is of the bad type.

The good advisor tells a lie $\left(m_{1}^{1}=0\right)$ to increase his reputation if

$$
-x_{1}^{1} \alpha_{3}+x_{2}^{1} \beta_{3}+x_{2}^{1} \eta_{3}>0
$$


where $x_{1}^{1}+x_{2}^{1}=1$ and

$$
\begin{aligned}
& \alpha_{3}=\frac{1}{2} \sum_{\epsilon=0}^{1}\left(P_{0, \epsilon}^{1,1}-P_{1, \epsilon}^{1,1}\right)\left[\rho_{\epsilon}\left\{-2 \gamma^{2}+\left(1-2 \gamma+2 \gamma^{2}\right)\left(P_{0, \epsilon}^{1,1}+P_{1, \epsilon}^{1,1}\right)\right\}+\nu_{\epsilon}\left(2 \gamma^{2}-\right.\right. \\
& \left.2 \gamma)\left(P_{0, \epsilon}^{1,1}+P_{1, \epsilon}^{1,1}-1\right)\right]>0, \\
& -\beta_{3}=f_{3}\left(\lambda_{1}^{1}, \lambda_{1}^{2}, \gamma, z, \rho, \nu\right)=f_{3}\left[g\left(\lambda_{2}^{1}\left(\lambda_{1}^{1}, 0,0\right)\right)-g\left(\lambda_{2}^{1}\left(\lambda_{1}^{1}, 1,0\right)\right)\right] \text { and } \\
& -\eta_{3}=f_{4}\left(\lambda_{1}^{1}, \lambda_{1}^{2}, \gamma, z, \rho, \nu\right)=f_{4}\left[g\left(\lambda_{2}^{1}\left(\lambda_{1}^{1}, 0,1\right)\right)-g\left(\lambda_{2}^{1}\left(\lambda_{1}^{1}, 1,1\right)\right)\right] .
\end{aligned}
$$

$\alpha_{3}$ explains the difference between the first advisor's first period payoff when he tells a lie and the payoff when he tells the truth. The first advisor believes that the second advisor has the same signal as him with probability $\frac{1}{2}$, i.e. $S_{1}^{2}=1$ with probability $\frac{1}{2}$. He also believes that the bad advisor sends the message 0 with probability $\rho$ if the signal of the second advisor is 1 and sends the message 0 with probability $\nu$ if the signal is 0 . Since the payoff of the good advisor is affected by both the action of the decision maker and the real state of the world, I need to separate the first period payoff as the case where the real state of the world is revealed as 0 or 1 .

$-\beta_{3}$ shows the difference between the first advisor's second period payoff which is determined by the value function of the first advisor when he tells a lie and the payoff when he tells the truth in the case where the real state of the world is revealed as 0 . When the first advisor believes that the second advisor observes the signal 1 , the updated belief of the decision maker that the second advisor is good is $\frac{1}{2}(1-\gamma)\left\{\rho \lambda_{2}^{2}\left(\lambda_{1}^{2}, 1,0\right)+(1-\rho) \lambda_{2}^{2}\left(\lambda_{1}^{2}, 0,0\right)\right\}$. Similarly, if the good advisor believes that the second advisor observes the signal 0 , the updated belief of the decision maker that the second advisor is good is $\frac{1}{2} \gamma\left\{\nu \lambda_{2}^{2}\left(\lambda_{1}^{2}, 1,0\right)+(1-\nu) \lambda_{2}^{2}\left(\lambda_{1}^{2}, 0,0\right)\right\}$.

$-\eta_{3}$ shows the difference between the first advisor's payoff when he tells a lie and the payoff when he tells the truth in the case where real state of the world is revealed as 1 . Since those expressions are very complicated, I use functional form $f$ and $g$ for simple explanation.

If the good advisor who meets the other bad advisor considers his second period as sufficiently more important, he sends the message the bad advisor is not biased towards, i.e. if

$$
x_{1}^{1}<\frac{\beta_{3}+\eta_{3}}{\alpha_{3}+\beta_{3}+\eta_{3}}=F_{G}\left(\lambda_{1}^{1}, \lambda_{1}^{2}, \gamma, z, \rho, \nu\right)<\frac{1}{2},
$$


the good advisor who observes the signal 1 sends the message 0 .

The area which guarantees the existence of the bad reputation effect increases with the probability that bad advisor sends the message 1 if his signal is 1 because of $\frac{\partial F_{G}\left(\lambda_{1}^{1}, \lambda_{1}^{2}, \gamma, z, \rho, \nu\right)}{\partial \rho}>0$, which means that $z$ decreases if $\rho$ increases.

\section{Appendix D}

The expressions in Appendix D is very similar as those in Appendix C. When the first advisor knows that the second advisor is also of the good type, the value function of the good advisor is

$$
\begin{aligned}
v_{G G}^{1}\left[\lambda_{2}^{1}, \lambda_{2}^{2}\right]= & -x_{2}^{1}\left(a_{2}-\omega_{2}\right)^{2} \\
= & \frac{1}{2} x_{2}^{1}\left[\gamma^{2}\left\{\left(P_{0,0}^{2,1}\right)^{2}+\left(P_{1,1}^{2,1}-1\right)^{2}\right\}\right. \\
& +\gamma(1-\gamma)\left\{\left(P_{0,1}^{2,1}\right)^{2}+\left(P_{1,0}^{2,1}\right)^{2}\right. \\
& \left.+\left(P_{1,0}^{2,1}-1\right)^{2}+\left(P_{0,1}^{2,1}-1\right)^{2}\right\} \\
& \left.+(1-\gamma)^{2}\left\{\left(P_{1,1}^{2,1}\right)^{2}+\left(P_{0,0}^{2,1}-1\right)^{2}\right\}\right] .
\end{aligned}
$$

If the good advisor who meets the other good advisor considers his second period as sufficiently more important, after observing the signal 1 he sends the message 0 .

By comparing the area which guarantees the existence of bad reputation effect in Appendix $\mathrm{C}$ with that in Appendix D, it is shown that the area which guarantees the existence of bad reputation effect when the good advisor meets the other bad advisor is bigger than the area which guarantees the existence of bad reputation effect when the good advisor meets the other good advisor.

\section{Appendix E}

Each type of advisor sends the message 0 in the first period because of the strong reputational concern to be perceived as good advisor. In the second period, if both advisors are good, the expected payoff of the decision maker is

$$
E_{G G}^{D M}=-\frac{1}{2} \sum_{\epsilon=0}^{1} \sum_{\zeta=0}^{1}\left[R_{\epsilon} R_{\zeta}\left(P_{\epsilon, \zeta}^{2,1}\right)^{2}+\left(1-R_{\epsilon}\right)\left(1-R_{\zeta}\right)\left(P_{\epsilon, \zeta}^{2,1}-1\right)^{2}\right]
$$

where $E_{T_{1} T_{2}}^{D M}$ represents the expected payoff of the decision maker if the first advisor is of the type $T_{1}$ and the second advisor is of the type $T_{2}$, and $R_{0}=\gamma$ 
and $R_{1}=1-\gamma$. It is because each good advisor sends the message which is the same as his signal and each advisor obtains the correct signal with probability $\gamma$. If the first advisor is good and the second advisor is bad, the expected payoff of the decision maker is

$$
E_{G B}^{D M}=-\frac{1}{2} \sum_{\epsilon=0}^{1} \sum_{\zeta=0}^{1}\left[R_{\epsilon} R_{\zeta}\left(P_{\epsilon, 1}^{2,1}\right)^{2}+\left(1-R_{\epsilon}\right)\left(1-R_{\zeta}\right)\left(P_{\epsilon, 1}^{2,1}-1\right)^{2}\right] .
$$

It is because bad advisor always sends the message 1 regardless of his signal. If both advisors are bad, the payoff of the decision maker is

$$
E_{B B}^{D M}=-\frac{1}{2}\left[\left(P_{1,1}^{2,1}\right)^{2}+\left(P_{1,1}^{2,1}-1\right)^{2}\right] .
$$

Similarly, the payoff of the decision maker in the second period when he has a single advisor is calculated. If the decision maker has good advisor, his expected payoff is

$$
E_{G}^{D M}=-\frac{1}{2} \sum_{\epsilon=0}^{1}\left[R_{\epsilon}\left(P_{\epsilon}^{2,1}\right)^{2}+\left(1-R_{\epsilon}\right)\left(P_{\epsilon}^{2,1}-1\right)^{2}\right]
$$

where $E_{T_{1}}^{D M}$ represents the expected payoff of the decision maker if advisor is of the type $T_{1}$. The payoff of the decision maker when he has bad advisor is

$$
E_{B}^{D M}=-\frac{1}{2}\left[\left(P_{1}^{2,1}\right)^{2}+\left(P_{1}^{2,1}-1\right)^{2}\right] .
$$

Since the decision maker believes both advisors are good, one advisor is good and the other advisor is bad, or both advisors are bad with the same probability, the payoff of the decision maker when he has two advisors is

$$
-\frac{1}{2}\left[\left(P_{0,0}^{1,1}\right)^{2}+\left(P_{0,0}^{1,1}-1\right)^{2}\right]+\frac{1}{3}\left[E_{G G}^{D M}+E_{G B}^{D M}+E_{B B}^{D M}\right] .
$$

The payoff of the decision maker when he has a single advisor is

$$
\frac{1}{2}\left[-\left(P_{0}^{1,1}\right)^{2}-\left(P_{0}^{1,1}-1\right)^{2}+E_{G}^{D M}+E_{B}^{D M}\right] .
$$

Under the case where each advisor considers his second period as sufficiently more important, since the expected payoff of the decision maker when he has a single advisor is greater than that when he has two advisors, it is better for the decision maker to consult only a single advisor. 


\section{REFERENCES}

Battaglini, M., "Multiple Referrals and Multidimensional Cheap Talk", Econometrica (2002), 1379-1401.

Battaglini, M., "Policy Advice with Imperfectly Informed Experts", Advances in Theoretical Economics (2004).

Crawford, V. P. and Sobel, J., "Strategic Information Transmission", Econometrica (1982), 1431-1451.

Ely, J. and Valimaki, J., "Bad Reputation", The Quarterly Journal of Economics (2001), 785-814.

Gibbons, R., "Game Theory for Applied Economists", Princeton University Press (1992).

Kreps, D., and Wilson, R., "Reputation and Imperfect Information", Journal of Economic Theory (1982), 253-279.

Krishna, V. and Morgan, J., "A Model of Expertise", The Quarterly Journal of Economics (2001), 747-775.

Levy, G. and Razin, R., "Multidimensional Cheap Talk", London School of Economics (2004).

Milgrom, P. and Roberts J., "Predation, Reputation and Entry Deterrence", Journal of Economic Theory (1982), 280-312.

Morris, S., "Political Correctness", Journal of Political Economy (2001), 231-264.

Olszewski, W., "Informal Communication", Journal of Economic Theory (2003).

Ottaviani, M. and Sorensen, P., "Professional Advice", Journal of Economic Theory (2005).

Park, In-uck, "Cheap Talk Referrals of Differentiated Experts in Repeated Relationship", Rand Journal of Economics (2005).

Selten, R., "The Chain-Store Paradox", Theory and Decision (1978), 127-159.

Sobel, J., "A Theory of Credibility", Review of Economic Studies (1985), $557-573$. 